\title{
Relativistic Interaction of Long-Wavelength Ultrashort Laser Pulses with Nanowires
}

\author{
Zhanna Samsonova, ${ }^{1,2, *}$ Sebastian Höfer, ${ }^{1}$ Vural Kaymak, ${ }^{3}$ Skirmantas Ališauskas, ${ }^{4}$ Valentina Shumakova, ${ }^{4}$ \\ Audrius Pugžlys, ${ }^{4}$ Andrius Baltuška, ${ }^{4}$ Thomas Siefke, ${ }^{5,6}$ Stefanie Kroker, ${ }^{6,7}$ Alexander Pukhov, ${ }^{3}$ Olga Rosmej, ${ }^{8,9}$ \\ Ingo Uschmann, ${ }^{1,2}$ Christian Spielmann, ${ }^{1,2}$ and Daniil Kartashov ${ }^{1}$ \\ ${ }_{1}^{1}$ Institute of Optics and Quantum Electronics, Abbe Center of Photonics, \\ Friedrich-Schiller-University Jena, Max-Wien-Platz 1, 07743 Jena, Germany \\ ${ }^{2}$ Helmholtz Institute Jena, Fröbelstieg 3, 07743 Jena, Germany \\ ${ }^{3}$ Institute for Theoretical Physics 1, Heinrich-Heine-University Düsseldorf, \\ Universitätsstraße 1, 40225 Düsseldorf, Germany \\ ${ }^{4}$ Photonics Institute, Vienna University of Technology, Gußhausstraße 27-29, 1040 Vienna, Austria \\ ${ }^{5}$ Institute of Applied Physics, Abbe Center of Photonics, Friedrich-Schiller-University Jena, \\ Albert Einstein Straße 15, 07745 Jena, Germany \\ ${ }^{6}$ Physikalisch-Technische Bundesanstalt, Bundesallee 100, 38116 Braunschweig, Germany \\ ${ }^{7}$ Laboratory for Emerging Nanometrology, Technical University Braunschweig, \\ Pockelsstraße 14, 38106 Braunschweig, Germany \\ ${ }^{8}$ GSI Helmholtz Centre for Heavy Ion Research, Planckstraße 1, 64220 Darmstadt, Germany \\ ${ }^{9}$ Goethe-University, Institute of Applied Physics, Max-von-Laue-Straße 1, \\ 60438 Frankfurt am Main, Germany
}

(Received 25 May 2018; revised manuscript received 14 February 2019; published 14 May 2019)

\begin{abstract}
We report on experimental results in a new regime of relativistic light-matter interaction employing midinfrared (3.9- $\mu \mathrm{m}$ wavelength) high-intensity femtosecond laser pulses. In the laser-generated plasma, electrons reach relativistic energies already for rather low intensities due to the fortunate $\lambda^{2}$ scaling of the kinetic energy with the laser wavelength. The lower intensity efficiently suppresses optical field ionization and creation of the preplasma at the rising edge of the laser pulse, enabling an enhanced efficient vacuum heating of the plasma. The lower critical plasma density for long-wavelength radiation can be surmounted by using nanowires instead of flat targets. Numerical simulations, which are in a good agreement with experimental results, suggest that $\approx 80 \%$ of the incident laser energy has been absorbed resulting in a longliving, keV-temperature, high-charge-state plasma with a density more than 3 orders of magnitude above the critical value. Our results pave the way to laser-driven experiments on laboratory astrophysics and nuclear physics at a high repetition rate.
\end{abstract}

DOI: 10.1103/PhysRevX.9.021029

\section{INTRODUCTION}

Solid density, (multi-)keV-temperature plasmas open new perspectives for realizing tabletop, high-brilliance $\mathrm{x}$-ray sources [1-3], laser-induced nuclear physics [4,5], and experiments on laboratory astrophysics $[6,7]$. Such plasmas can be generated when relativistically intense, high-temporal-contrast femtosecond laser pulses interact with solids. In the relativistic regime of laser-matter interaction, the velocity of the laser-field-driven free electrons approaches the speed of light. Quantitatively, it is reached if the normalized

\footnotetext{
* Corresponding author. zhanna.samsonova@uni-jena.de

Published by the American Physical Society under the terms of the Creative Commons Attribution 4.0 International license. Further distribution of this work must maintain attribution to the author(s) and the published article's title, journal citation, and DOI.
}

Subject Areas: Plasma Physics

vector potential $a_{0} \geq 1$, where $a_{0}=0.85 \times 10^{-9} \sqrt{I} \lambda$, with $I$ being the laser peak intensity in watts per square centimeter and $\lambda$ the laser wavelength in microns. A common way to enter the relativistic regime is to increase the laser intensity. For a given laser pulse energy, higher intensities can be reached easier with short-wavelength laser sources by entering the so-called $\lambda^{3}$ regime, defined by focusing the shortest possible pulses (given by the length of a single cycle) to the diffraction limited spot given by the wavelength [8]. However, such single-cycle, short-wavelength ultraintense pulses will only interact with an extremely small volume of plasma. It is also worth mentioning that pulse compression down to a single cycle becomes extremely challenging with a shortening of the laser wavelength.

Alternatively, $a_{0}$ can be more efficiently increased using long-wavelength laser sources. In this case, highly relativistic interaction can be realized with moderate laser-pulse energies paving the way to high-repetition-rate experiments in a relatively large volume. This new regime of relativistic, 
ultrashort-pulse laser-solid interaction is inaccessible so far, because $\mathrm{CO}_{2}$ lasers, the only high-energy, midinfrared (mid-IR) laser sources available, emit pulses with a duration $>1$ ps $[9,10]$. At this timescale, the hydrodynamic expansion during the pulse prevents plasma densities above the critical value. Because of the recent progress in the development of high-energy optical parametric chirpedpulse amplification (OPCPA) femtosecond laser systems, nowadays femtosecond laser pulses of terawatt peak power level in mid-IR spectral range are available [11,12].

The requirement of high temporal contrast of a relativistically intense, ultrashort laser pulse is one of the necessary conditions to achieve high density and sharp (on the laser wavelength scale) spatial gradients in plasmas. A sharp gradient of the density enables an efficient vacuum heating mechanism of the laser energy absorption [13-17], thus ensuring a high temperature of generated dense plasmas. For high power laser systems in the near-IR spectral range, commonly used for relativistic laser-solid interactions, the requirement of the high temporal contrast inevitably invokes frequency doubling of the output radiation, reducing the ionization threshold to a few- or even single-photon absorption. Significant reduction of the ionization rate at mid-IR frequencies in comparison to near-IR-visible-UV laser sources [18], together with long wavelength in mid-IR laser pulses, substantially relax the problem of preplasma formation and achieving sharp spatial gradients of the plasma density. In particular, an advantage of mid-IR femtosecond driver pulses, compared to near-IR pulses, for the efficiency of vacuum heating of solid target under nonrelativistic interaction, resulting in a much higher yield of the $K_{\alpha}$ emission from a $\mathrm{Cu}$ tape, was recently demonstrated $[19,20]$.

Here, we report for the very first time, to the best of our knowledge, experimental results on a new regime of relativistic laser-solid interaction drawing on the combination of midrared wavelength, high peak power, high temporal contrast femtosecond laser source, and advanced nanostructured silicon samples as the target. The measured characteristic line and bremsstrahlung $\mathrm{x}$-ray spectra, together with numerical simulations of the laser-plasma interaction and plasma emission, based on the particle-in-cell (PIC) [21] and collisional radiative population kinetics [22] codes, reveal that the nanowire morphology allows overcoming a problem of the unfavorable scaling of the critical electron density with the wavelength $n_{\mathrm{cr}}\left[\mathrm{cm}^{-3}\right] \approx 10^{21} / \lambda^{2}[\mu \mathrm{m}]$. The generated plasmas have solid density, corresponding to an unprecedented $>10^{3} n_{\mathrm{cr}}$ at the driving laser wavelength.

\section{EXPERIMENTAL SETUP}

The experiments were carried out at the high-energy OPCPA laser system delivering 90-fs laser pulses at $3.9 \mu \mathrm{m}$ idler wavelength with an energy on the target up to $25 \mathrm{~mJ}$ at $20-\mathrm{Hz}$ repetition rate $[11,12]$. The beam is focused by an off-axis parabolic mirror to a $12-\mu \mathrm{m}$ (FWHM) focal spot under $45^{\circ}$ angle with respect to the target's normal [see Fig. 1(a)]. The pulses are characterized by the SHG FROG measurements and the focal beam size is measured by the knife-edge scan method. The maximum peak intensity at the target is estimated to be $10^{17} \mathrm{~W} / \mathrm{cm}^{2}$, resulting in the maximum value of the relativistic parameter $a_{0} \approx 1$.1. All the measurements were conducted in a single-shot regime.

In conventional chirped-pulse-amplification laser systems based on the laser principle of amplification, the temporal contrast at the nanosecond-picosecond timescale is determined by the level of the amplified spontaneous emission and amplification of any parasitic prepulses [23]. For OPCPA, the temporal structure of the output pulse is mainly defined by the emission of superfluorescence having the duration of the pump laser pulse ( $80 \mathrm{ps}$ for our system). To get an estimate of the temporal contrast in our experiments, the seed signal beam is blocked to measure the energy of the superfluorescence radiation. The background signal is below the milliwatt level which is the sensitivity threshold of the used detector for the $20-\mathrm{Hz}$ repetition rate. This number has to be compared with the amplified idler signal at the $25-\mathrm{mJ}$ output energy resulting in 500-mW measured average power. Thus, the energy in the $\sim 80$-ps superfluorescence pulse is below $50 \mu \mathrm{J}$ and the corresponding peak intensity of $\sim 10^{11} \mathrm{~W} / \mathrm{cm}^{2}$ at $3.9 \mu \mathrm{m}$ is too low to generate any significant amount of plasma before the arrival of the main pulse.

The targets represent single crystalline $\mathrm{Si}$ nanowire (NW) arrays [Fig. 1(b)] on a silicon substrate, transparent
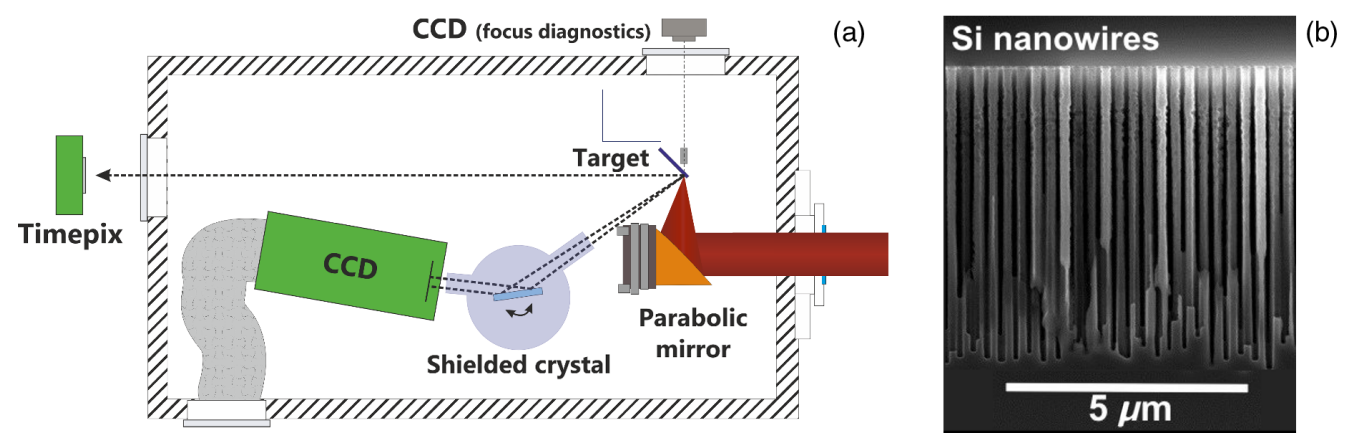

FIG. 1. (a) Experimental setup. (b) Scanning electron microscope (SEM) image of the Si nanowire target. 
in the broad spectral range of 1.2-7 $\mu \mathrm{m}$. The details of the target fabrication are given in Ref. [24]. Briefly, 500- $\mu \mathrm{m}-$ thick Si substrates are coated with a chromium layer, followed by an electron-beam sensitive resist. The periodic patterns are created during the electron-beam lithography with character projection. After the development of the resist, reactive ion etching is carried out to transfer the structure to the chromium layer and subsequently to the silicon substrate. Finally, residuals of resist and chromium layer are removed using wet chemical etching. The resulting density of the Si NWs is about $35 \%$ solid material density, which means that a focal spot of $12 \mu$ m contains roughly 1600 NWs. Each NW is $6 \mu \mathrm{m}$ long and has a diameter of $200 \mathrm{~nm}$. The spacing between the NWs is about $100 \mathrm{~nm}$. The reference target is a $500-\mu \mathrm{m}$-thick polished $\mathrm{Si}$ wafer. All samples have an area $\sim 1 \mathrm{~cm}^{2}$ allowing several tens of shots per sample with pretty good reproducibility of the measured $\mathrm{x}$-ray spectra and hard $\mathrm{x}$-ray spectra due to low (below 5\%) shot-to-shot fluctuations of the laser energy.

The experimental diagnostics include a high-resolution x-ray crystal spectrometer for the range of 1.7-2.1 keV covering $K$-shell characteristic emission lines from $\mathrm{Si}$ (from $K_{\alpha}$ to $K_{\beta}$ ) for all charge states up to H-like, and a detector for characterizing the hard x-ray-gamma-ray bremsstrahlung emission spectrum. The crystal spectrometer is based on a flat potassium acid phthalate crystal combined with a cooled back-illuminated x-ray CCD camera. The hard x-ray and gamma-ray spectra in a broad energy range are measured with a Timepix detector, based on a CMOS pixel readout chip working in a single photon counting mode. A 1-mm-thick CdTe sensor chip enables the registration of photons with energies up to $800 \mathrm{keV}$ [25]. The focusing parabola, the targets, and the spectrometer are placed in a vacuum chamber pumped below $10^{-4}$-mbar pressure. The Timepix detector is located in ambient air $\sim 5 \mathrm{~m}$ away from the target and oriented at $45^{\circ}$ to the target normal observing it through a $230-\mu \mathrm{m}$ thick Kapton window.

\section{EXPERIMENTAL RESULTS}

The spectra of the characteristic line emission from the polished and nanowire targets are shown in Fig. 2(a). Under conditions of our experiment the efficiency of the $K_{\alpha}$ emission from weakly ionized Si ("cold" emission), is essentially the same from both morphologies. This result is strikingly different from the results known in the nonrelativistic regime of interaction [26-28] and suggests that in the relativistic limit nanostructure arrays have no advantages in comparison to the flat surface in terms of the efficiency of the characteristic line emission from weakly charged states.

In contrast to the characteristic emission from $\mathrm{Si}^{+}-\mathrm{Si}^{4+}$ contributing to the $K_{\alpha}$ line, Fig. 2(a) demonstrates a dramatic increase in the efficiency of the line emission from highly charged ion species for the nanowire arrays in comparison to the flat surface. Emission from Li-like $\left(\mathrm{Si}^{11+}\right)$, He-like $\left(\mathrm{Si}^{12+}\right)$ and $\mathrm{H}-$ like $\left(\mathrm{Si}^{13+}\right)$ ions, such as $\mathrm{He}_{\alpha}, \mathrm{He}_{\beta}$, and $\mathrm{Ly}_{\alpha}$ is detected from the nanowire arrays only. Note that emission of $K L$ transitions from $\mathrm{Si}^{11+}$ overlaps with $K_{\beta}$ emission line of $\mathrm{Si}^{+}$ions. Though their maxima are slightly shifted with respect to each other, this shift is not resolved by our spectrometer. It is known that the ratio of the intensities of $K_{\alpha}$ and $K_{\beta}$ emission lines in $\mathrm{Si}$ is about 50:1 [29,30], which matches well to the measured ratio of $42: 1$ in the emission spectrum from the polished target [Fig. 2(a)]. In contrast, this ratio for the nanowires is $6: 1$ suggesting a dominant contribution of the $K L$ transition emission from the Li-like $\mathrm{Si}$ charge state in the spectral range $1.83-1.85 \mathrm{keV}$ [the spectral range covering $\mathrm{Si}^{11+}$ emission marked in Fig. 2(a)]. Because of the relatively high stability of the laser pulse energy, shot-to-shot variations in the intensity of He-like and H-like
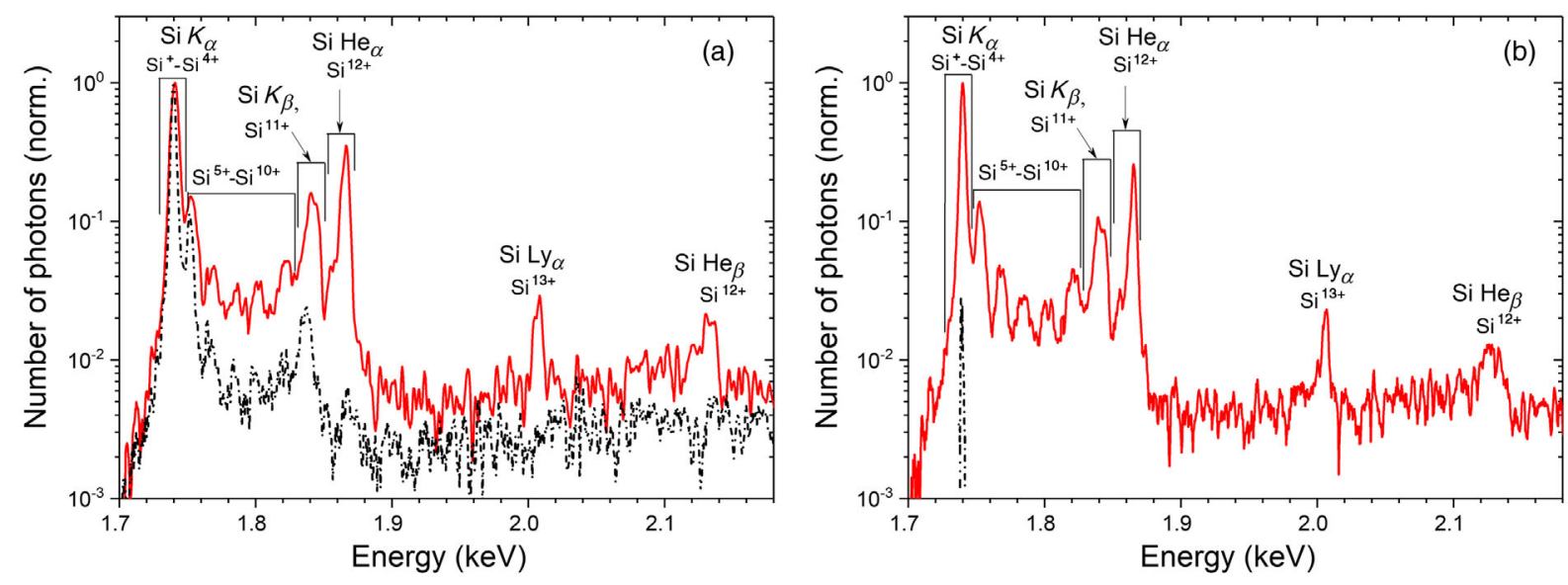

FIG. 2. (a) Spectrum of $K$-shell line emission from the polished (black dash-dotted line) and the nanowire array (red solid line) Si targets in the logarithmic scale measured at (a) $45^{\circ}$ and (b) $0^{\circ}$ incidence angles (with respect to the target's normal). 
emission lines are within $20 \% \mathrm{rms}$, whereas variations in the intensity of "cold" $K_{\alpha}$ emission are within $14 \% \mathrm{rms}$.

The same measurements are also performed for $0^{\circ}$ incidence angle (normal to the target surface) [Fig. 2(b)]. In this case, the intensity of the $K_{\alpha}$ emission line of $\mathrm{Si}^{+}$ions from the polished target drops by more than an order of magnitude, whereas emission at characteristic lines of highcharge-state ions is not detected at all. Such behavior can be explained assuming the Brunel mechanism of absorption, for which the absorption efficiency approaches zero when the laser polarization becomes parallel to the target surface [13]. Also, the results for "cold" $K_{\alpha}$ emission are in a good agreement with the results published in Ref. [19] for a $\mathrm{Cu}$ target and explained by the Brunel mechanism of electron heating. At the same time, the spectrum obtained with the NW target remains essentially unchanged. This insensitivity of nanowire morphology to the orientation of the laser polarization is observed in the experiments on interactions in the nonrelativistic regime [31] and can be explained by the fact that there is always a significant component of the laser field perpendicular to the local surface for this target morphology. In the following discussions, we focus on the results obtained in the interaction under the $45^{\circ}$ incidence angle.

Similar to the measurements of "cold" $K_{\alpha}$ emission, the high-energy bremsstrahlung spectra show almost no difference in the flux up to $140 \mathrm{keV}$ for the flat and nanostructured targets (Fig. 3). By applying an exponential fit to the hard x-ray photon distribution [32], for both target morphologies, we estimate approximately the same hot electron temperature $T_{e} \approx 26 \mathrm{keV}$ for the electron fraction responsible for the emission of the photons in the energy range 40-140 keV. The "hot" electron temperature

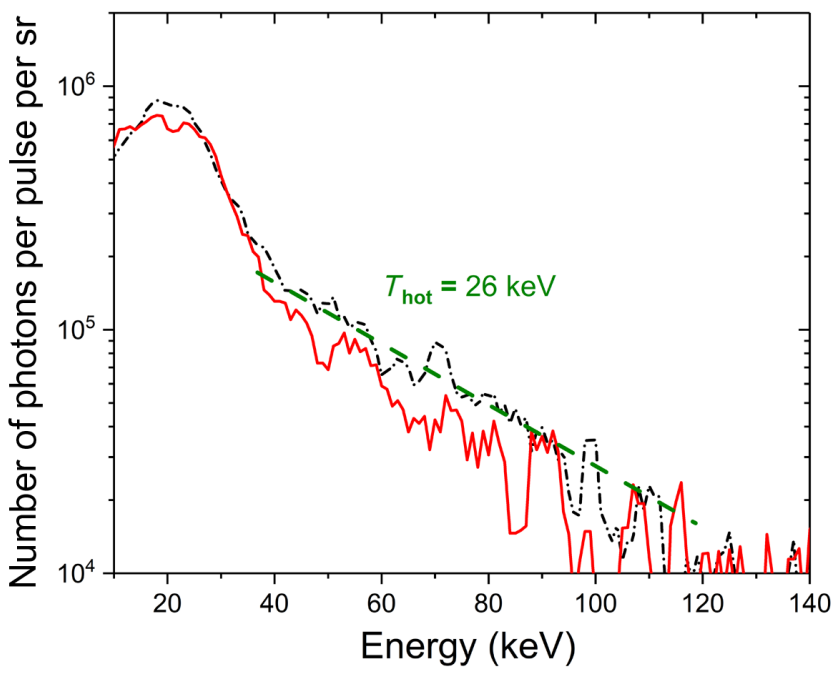

FIG. 3. Hard x-ray emission spectrum measured from the polished Si target (black dash-dotted line) and the nanowire array (red solid line). The green dashed line is the exponential fit providing an estimate of the hot electron temperature $T_{\text {hot }}=26 \mathrm{keV}$. estimated from the hard $\mathrm{x}$-ray spectrum in Fig. 3 is a temperature of the electron fraction heated during the laser pulse via the Brunel mechanism [13-17]. The general structure of the electron energy distribution function (EEDF) for a relativistic laser-plasma interaction is up to now an open question because numerical simulations in the entire energy range, from the relatively cold major part of EEDF to the ultrafast relativistic tail controlling ion acceleration, are extremely challenging. Also, it is sensitive to several parameters like the spatial gradient of the plasma density and $a_{0}$ [13-17]. However, from the general physical point of view, it is reasonable to expect that the EEDF should consist of at least three fractions of electrons. The first one, commonly termed as "hot" electrons in the particle acceleration community, constitutes only a tiny fraction of the total amount of electrons. They are accelerated and pushed out by the pondermotive force in the laser pulse and leave the target forming the charge separation sheet at the surface [33,34]. The second fraction, which we call "hot" in this paper, is forming an energetic tail of the EEDF due to the Brunel heating mechanism in the laser pulse. According to the PIC simulations (see below), under the conditions of our experiments this fraction contains less than $1 \%$ of the total amount of electrons. Finally, the third fraction, containing the absolute majority of electrons, has the bulk electron temperature which can be estimated from simulations of the measured line-emission spectra (see below). Examples of such a threetemperature EEDF are described, e.g., in Refs. [35,36].

\section{DISCUSSIONS}

To get insight into the physics of processes occurring at different timescales under the conditions of our experiments, we perform two sets of numerical simulations. First, we use particle-in-cell (PIC) simulations using the VIRTUAL LASER LAB code [21] to retrieve the parameters of plasma and their dynamics under relativistic interaction of ultra-high-temporal contrast ultrashort laser pulses with Si nanowire arrays. The obtained temperature for hot and bulk electrons and their temporal evolution is used for calculating the line-emission spectra using the FLYCHK atomic kinetic code [22]. For FLYCHK we assume a constant ion density of $5 \times 10^{22} \mathrm{~cm}^{-3}$ implying that the NWs stay intact over the simulation time of $1.5 \mathrm{ps}$, while the electron density evolution is considered by the code.

The nanowire PIC simulation is done in a box with the size $17.55 \mu \mathrm{m} \times 0.3 \mu \mathrm{m} \times 0.3 \mu \mathrm{m}$. The grid steps are $6.65 \mathrm{~nm} \times 5.77 \mathrm{~nm} \times 5.77 \mathrm{~nm}$ and the time step is 5.2 as. The flat target simulation is done in a $82.73 \mu \mathrm{m} \times$ $137.39 \mu \mathrm{m}$ box with grid steps $73.86 \mathrm{~nm} \times 73.86 \mathrm{~nm}$ and a time step of 1.3 as. The laser pulse has a Gaussian temporal profile of 90-fs duration and a normalized vector potential of $a_{0}=1.05$. It is modeled as a plane wave in the nanowire simulation with periodic boundary conditions in transverse 
direction for both particles and fields. In the flat target case the laser is focused to a focal spot size of $12 \mu \mathrm{m}$ (FWHM in diameter) with a Gaussian transverse profile, having an angle of incidence of $45^{\circ}$. In both simulations the material of the targets is non-preionized silicon which is assumed to be initially cold. To take into account ionization of the material both optical field and collisional ionization modules are included.

The spatial distribution of the bulk electron temperature along the nanowires at different moments of time calculated with the PIC code is shown in Fig. 4(a). It reaches the maximum temperature of $\approx 600 \mathrm{eV}$ within an approximately $1-\mu \mathrm{m}$-thick layer at the tip of the wires during the interaction with the laser pulse. After the laser pulse ( $t=300 \mathrm{fs}$ after its peak), the entire wire volume is heated almost homogeneously to approximately $50 \mathrm{eV}$.

The spatial distribution of the electron density in the wire volume is shown in Fig. 4(b). It reaches the maximum value $n_{e} \sim 6 \times 10^{23} \mathrm{~cm}^{-3}$, corresponding to fully ionized $\mathrm{Si}$ atoms up to $\mathrm{Si}^{12+}$ (He-like) charge state, within the hot $\approx 1-\mu$ m-thick upper layer of the wires on the timescale of the laser pulse duration. After the laser pulse $(t=300 \mathrm{fs}$ after its peak), the main volume of the wires is ionized with the electron density $\sim 3 \times 10^{23} \mathrm{~cm}^{-3}$ and thermalizes to the electron temperature of about $30 \mathrm{eV}$. Considering the value
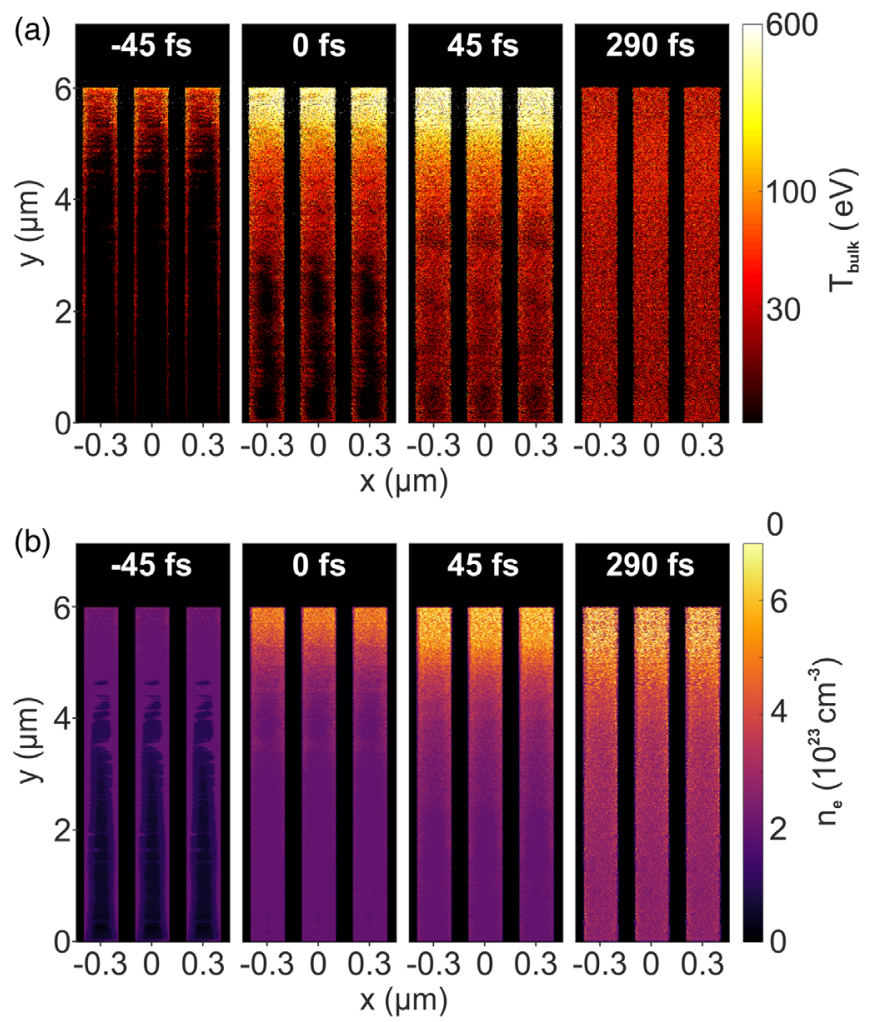

FIG. 4. Spatial distribution of (a) the electron temperature and (b) electron density in $\mathrm{Si}$ nanowires retrieved from the PIC simulations. The zero moment of time corresponds to the moment of the peak intensity arrival at the tip of the wires. of the critical density $n_{c} \approx 7 \times 10^{19} \mathrm{~cm}^{-3}$ at $3.9-\mu \mathrm{m}$ laser wavelength, the simulations suggest that plasma as dense as about $\approx 10^{4} n_{c}$ is generated under the conditions of the experiment. Our simulations show also that the wires stay intact and high plasma density is maintained on the timescale of at least $2.9 \mathrm{ps}$. This timescale is significantly longer than a femtosecond timescale of wire explosion predicted for shortlaser wavelength experiments [37-39]. Simulations suggest that at large values of $a_{0}$ pinching of the electron current driven by a relativistically intense laser pulse along the wire can effectively heat electrons and increase plasma density, which largely speeds up the hydrodynamic expansion of the plasma at $a_{0} \geq 1$ [39]. The amplitude of the fields responsible for the pinch effect scales with the laser wavelength as $\lambda^{-1}$. Further, the number of forward-driven electrons is proportional to the critical density associated with the laser wavelength that scales as $\lambda^{-2}$. Simple estimates show that for long-wavelength laser sources, the laser-induced electron currents are too low to cause a significant pinching effect. Therefore, an unprecedentedly long lifetime of overdense plasma predicted under the condition of our experiment is due to the long laser wavelength.

The $\approx 1 \mu \mathrm{m}$ depth of the high-density and hightemperature plasma region is determined by the penetration depth of the laser pulses into the wire array which, in turn, is limited by the absorption. The simulations predict $76 \%$ absorption efficiency of the laser energy which is concentrated mostly within the $\approx 1 \mu \mathrm{m}$ layer (for the given 20 -mJ laser pulse energy) due to an extremely high $n_{e} / n_{c}$ ratio.

High temperature and density of plasma enabled by NW morphology play a key role in reaching a high-charge state and efficient emission from He-like and $\mathrm{H}$-like ions. Note that simulations based on the Popov-Perelomov-Terentiev ionization model [40] show that the optical field ionization for the experimental field parameters is capable of ionizing $\mathrm{Si}$ atoms up to a $\mathrm{Si}^{4+}$ charge state only, therefore higher charge states are the result of collisional plasma heating and generated via the electron impact ionization mechanism. The evolution of the charge states, populations of different excited ion states, and the corresponding emission spectrum are simulated using the kinetic FLYCHK code with the temporal history of the bulk and hot electron temperatures derived from the PIC simulations. The large volume of the dense plasma predicted by the PIC simulations results in large wavelength-dependent plasma opacity, causing strong reabsorption of the generated emission and, therefore, influencing the emission line ratio. To include the effect of opacity, the effective thickness of the emitting plasma layer is used as a fitting parameter to match the simulated spectra to the measured ones. The effective thickness of the emitting plasma layer providing the best match between the simulated and the measured $\mathrm{x}$-ray spectra is found to be $\approx 0.5 \mu \mathrm{m}$. The temporal evolution of the bulk and hot electron temperatures averaged over the volume of the upper $0.5 \mu \mathrm{m}$ layer of 

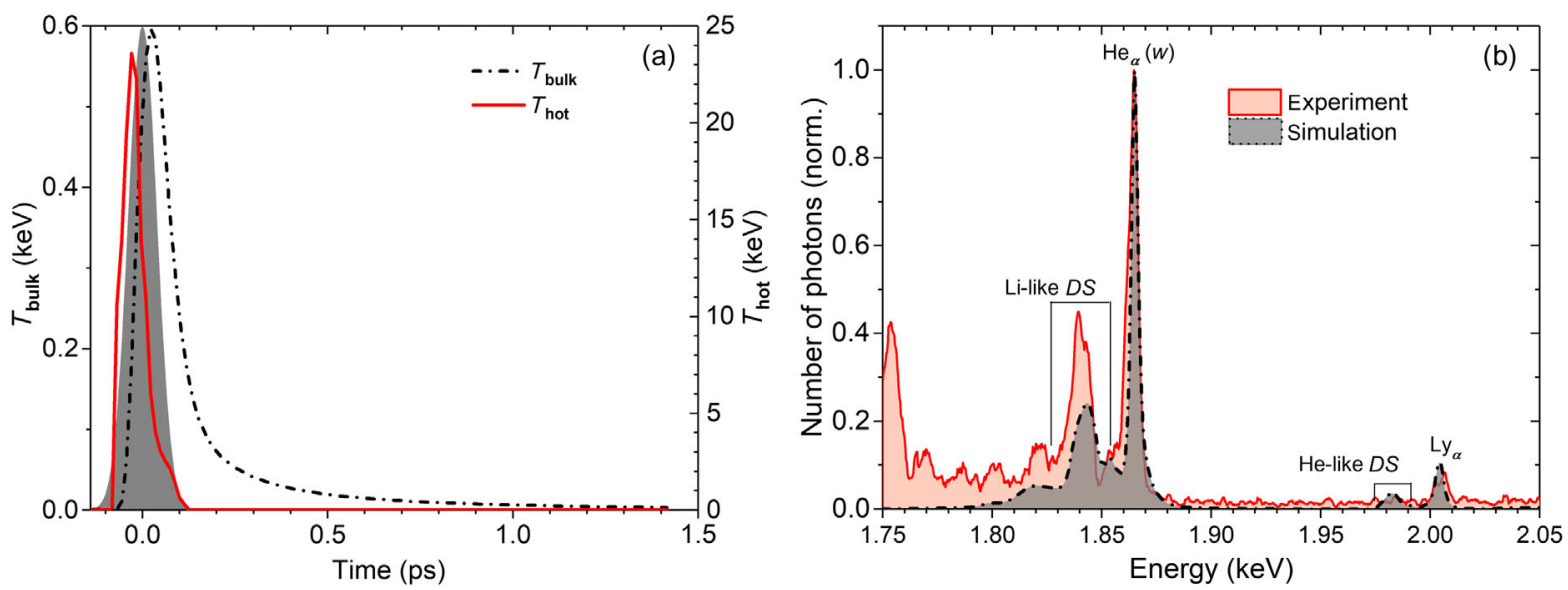

FIG. 5. (a) The temporal evolution of the temperature of the "hot" (red line) and the bulk (black dash-dotted line) electron fractions averaged over the upper $0.5 \mu \mathrm{m}$ length of the wire. The gray shaded curve is the temporal profile of the laser intensity. (b) The measured (red line) and the simulated (black dash-dotted line) spectra (time integrated).

wires is shown in Fig. 5(a). The maximum average bulk temperature of $\approx 0.6 \mathrm{keV}$ is reached by the end of the laser pulse and cools down with 100 fs time constant. The hot electron temperature reaches its maximum of about $25 \mathrm{keV}$ within the laser pulse duration and vanishes as soon as the laser pulse is off. The calculated maximum is in very good agreement with the hot electron temperature estimated from the measured hard x-ray spectra (see Fig. 3). The simulated emission spectrum is shown in Fig. 5(b) and demonstrates an excellent agreement with the most energetic part of the experimental spectrum, consisting of $\mathrm{He}$ - and $\mathrm{H}$-like $\mathrm{Si}$ emission lines and emitted at the maximum intensity region in the focal distribution. Note that the simulation result is quite sensitive to the changes of the input parameters and varying the electron temperature by more than $10 \%$ cannot be simply compensated by a reasonable density adjustment. Therefore, we conclude that the used set of plasma parameters indeed fits well to the experimental findings. It is worth mentioning also that the temporal evolution of the electron density during the laser pulse, calculated by the FLYCHK code, agrees well with the evolution of the electron density in the PIC simulations averaged over the $0.5-\mu \mathrm{m}$ upper layer. After the laser pulse, the electron density predicted by the FLYCHK simulations drops by a factor of 2 within approximately 200 fs due to efficient recombination processes, which are not included in the PIC simulations.

In a next step, we want to understand the temporal structure of the emitted x-ray radiation. FLYCHK simulations allow us to get insight into the temporal evolution of the charge states and the emitted spectrum [Fig. 6(a)], which are governed by the evolution of the bulk electron temperature and collisional and radiative rates. Formation of He-like and $\mathrm{H}$-like Si ions occur due to the collisional
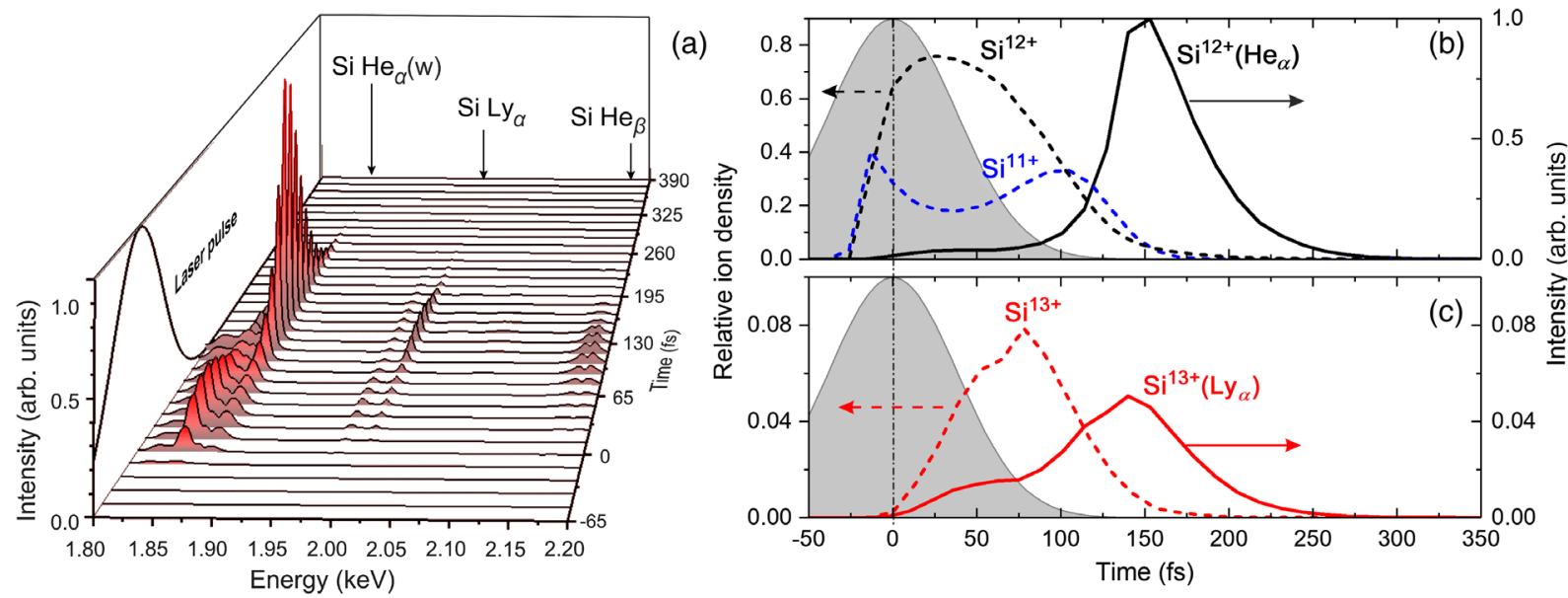

FIG. 6. (a) The temporal evolution of the $K$-shell emission in the spectral region $1.8-2.2 \mathrm{keV}$. (b), (c) Time-dependent density of $\mathrm{Si}^{11+}$ (dashed blue line), $\mathrm{Si}^{12+}$ (dashed black line), and $\mathrm{Si}^{13+}$ (dashed red line) ions; intensity of $\mathrm{He}_{\alpha}$ (solid black line) and Ly ${ }_{\alpha}($ solid red line) emission. The densities normalized to the density of neutral atoms. The shaded curve is the normalized laser pulse intensity. 
ionization of $\mathrm{Si}^{11+}$ and $\mathrm{Si}^{12+}$, respectively. Ionization of the $L$ shell up to the charge state $\mathrm{Si}^{12+}$ takes place at the rising edge of the bulk electron temperature (about $25 \mathrm{fs}$ after the peak of the laser pulse intensity) [Fig. 6(b)]. Meanwhile, ionization of the $K$ shell requires electrons with a kinetic energy of more than $2 \mathrm{keV}$ (ionization bottleneck). Since the rates of the collisional processes in plasma drop exponentially with an increasing ratio of the binding energy $E_{\text {bin }}$ to the bulk electron temperature $T_{\text {bulk }}, \mathrm{Si}^{13+}$ ions are generated with a delay in respect to the appearance of $\mathrm{Si}^{12+}$ and approaching their highest density at roughly 75 fs [Fig. 6(c)]. After reaching their maxima, both ion densities decrease following the $T_{\text {bulk }}$ behavior. The comparison of ion fractions obtained from time-dependent FLYCHK simulations with a steady-state distribution at $T_{\text {bulk }}=$ $600 \mathrm{eV}$ shows that the maximal value of the $\mathrm{Si}^{13+}$ fraction [8\% reached at $\sim 75$ fs, Fig. 6(c)] is one order of magnitude lower than the value obtained in the steady temperature case, while the maximal value of the $\mathrm{Si}^{12+}$ fraction is twice higher $[80 \%$ reached at $\sim 25$ fs, Fig. 6(b)]. Therefore, simulations suggest the transient character of the plasma under our experimental conditions.

The $K$-shell radiation $\left(\mathrm{He}_{\alpha}\right.$ and $\left.\mathrm{Ly}_{\alpha}\right)$ arises mainly from the collisional excitation of the $K$-shell electrons followed by the radiative decay back into the ground state. The timescale of the buildup of the $\mathrm{Si}^{12+}$ and $\mathrm{Si}^{13+}$ ion densities and the timescale of the corresponding electron impact excitations are similar. Therefore, the temporal evolution of the $\mathrm{He}_{\alpha}$ and $\mathrm{Ly}_{\alpha}$ line emission starts almost simultaneously to the corresponding ion density. Neglecting effects of opacity, the maximum of the emission is delayed by $\sim 25 \mathrm{fs}$ for $\mathrm{He}_{\alpha}$ and $\sim 50$ fs for $\mathrm{Ly}_{\alpha}$ with respect to the maximum of the corresponding ion density in accordance with the radiative decay times [22]. However, the $\mathrm{He}_{\alpha}$ line resembles the second maximum in the emission related to the recombination channel of the population from H-like ions. The time-dependent opacity strongly modifies the temporal profile of the line emission resulting in $\sim 50$ fs at FWHM pulse at the $1.865-\mathrm{keV} \mathrm{He}_{\alpha}$ transition and $\sim 150 \mathrm{fs}$ at
FWHM pulse at the 2-keV $\mathrm{Ly}_{\alpha}$ transition [Figs. 6(b) and 6(c)]. The expected shorter duration of the $\mathrm{He}_{\alpha}$ emission is a consequence of higher opacity of the plasma at this emission wavelength. Overall, the ultrashort duration of the generated x-ray emission in relativistic interaction of ultrahigh contrast, ultrashort laser pulses with solids is intrinsically related to the transient character of highdensity plasma. This transient nature of the plasma evolution has to be taken into account when estimating the timescale of radiation cooling processes and determining conditions for new regimes where the plasma cooling caused by plasma self-radiation happens faster than hydrodynamic expansion [41]. High electron density enables ultrafast collisional pumping. At the same time, high ionic density (in the corresponding charge state) leads to strong absorption of the emitted line radiation (opacity) and the reemission of the absorbed photons is strongly suppressed due to the collisional deexcitation by free electrons. Thus, a delay between the maximum of the laser pulse and maximum of the $K$-shell radiation is expected as it is demonstrated in Fig. 6. Therefore, we conclude that the high density and temperature of plasma, dynamically changing at subpicosecond timescale, in combination with (dynamically changing) effects of opacity are determining the femtosecond duration of the $\mathrm{x}$-ray line emission in contrast to picosecond duration estimated from assumptions of optically thin plasma under steady-state conditions [41].

Finally, simulations are carried out for spectra measured from a polished $\mathrm{Si}$ wafer to understand the differences in the emission for different morphologies of the samples. The results of the spatial and temporal evolution of the bulk electron temperature and density obtained in PIC simulations are presented in Fig. 7. As follows from Fig. 7(b), the plasma density for flat targets can be as high as $3 \times 10^{23} \mathrm{~cm}^{-3}$ which is comparable to the density calculated for nanowires [Fig. 4(b)]. However, the maximum bulk electron temperature is only $50 \mathrm{eV}$ and reached just within an approximately $100-\mathrm{nm}$ thin layer near the surface. Compared to nanowires, the substantially lower
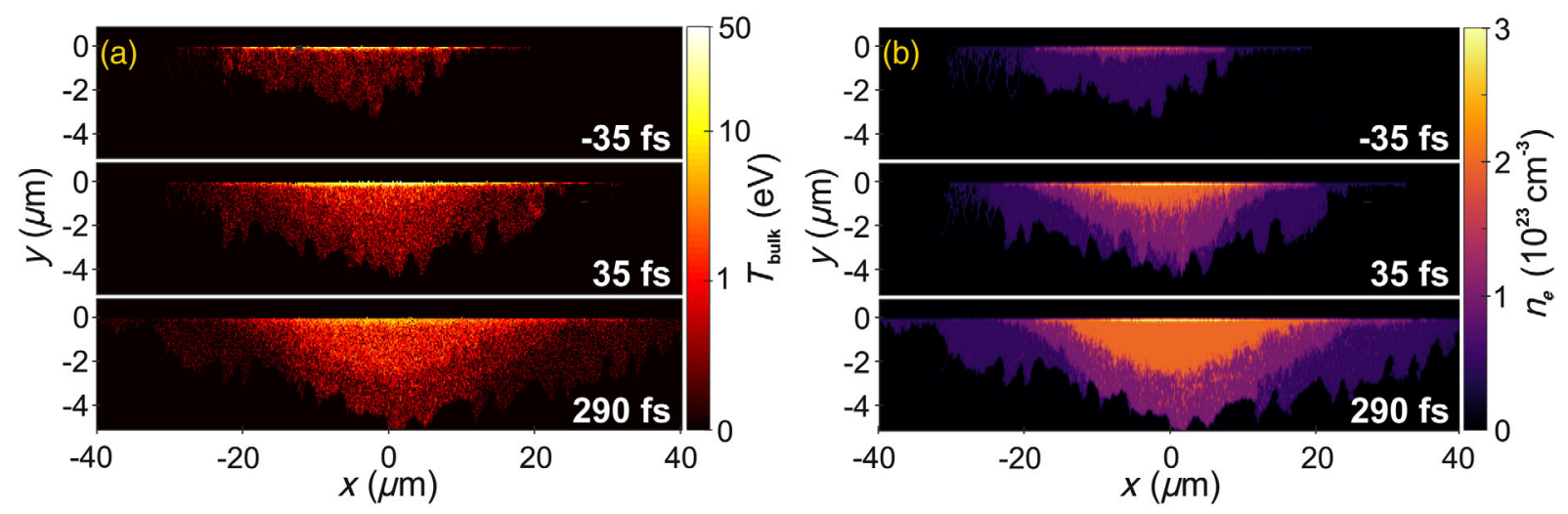

FIG. 7. The results of PIC simulations for the spatial distribution of the (a) bulk electron temperature and (b) electron density at the flat Si target. The zero moment of time corresponds to the moment of the peak intensity arrival at the surface boundary. 
electron temperature is the consequence of a strong $(\approx 98 \%)$ reflection of the laser energy from the overcritical plasma. Simulations of the spectra by FLYCHK fail for such dense and low-temperature plasma. The observed spectra can be understood by extending the concept of Brunel electron heating [13], into the relativistic regime [20]. With this mechanism, electrons are efficiently accelerated near the critical density and the subsequent collision creates holes in the $K$ shell of neutral $\mathrm{Si}$ atoms followed by cold $K_{\alpha}$ emission. Using the same long-wavelength laser system as in our experiments, an enhanced conversion efficiency for $K_{\alpha}$ radiation from $\mathrm{Cu}$ foil has been demonstrated in [19]. Also, we assume that the weak emission in the range of 1.747-1.827 keV [Fig. 2(a)], observed for all the targets and originating from intermediate charge states of $\mathrm{Si}$ (up to $\mathrm{Si}^{10+}$ ), is generated due to innershell collisional ionization by energetic electrons emerging in the Brunel heating process. The difference in the yield of this emission between the nanowires and the bulk target originates from the difference in the bulk electron temperature since mainly the bulk electrons are responsible for the evolution of the charge states in the plasma.

\section{CONCLUSIONS}

Relativistic interaction of high temporal contrast, femtosecond laser pulses with solids is investigated experimentally and numerically in a new regime drawing on long-wavelength (mid-IR) laser radiation. In this interaction regime, profiting from favorable $\lambda^{2}$ scaling of electron energies with the laser wavelength rather than linear scaling with intensity, the laser field ionization and creation of the preplasma on the rising edge of the laser pulse are strongly suppressed. As a consequence, the maximum of the laser pulse interacts with solid matter and ionization has predominantly a collisional character. Comparing the experimentally measured $x$-ray emission spectra with the results of numerical simulations confirms the importance of nanowire arrays as a solid target for overcoming the problem of the low critical plasma density at mid-IR wavelengths enabling very efficient absorption of the laser pulse energy $(\approx 80 \%)$. As a result, plasma with an electron density up to $6 \times 10^{23} \mathrm{~cm}^{-3}$ is generated, which is a record high density if it is measured in critical density values and is more than $10^{3}$ times the critical density. The high electron density of the created plasmas ensures high collision rates that govern ionization of the target material up to the H-like charge state $\left(\mathrm{Si}^{13+}\right)$ and $0.6 \mathrm{keV}$ peak bulk electron temperature.

Using NW targets and mid-IR laser pulses we are able to generate solid density plasmas with keV-level bulk temperatures using only 20 -mJ energy per laser pulse, in contrast to Joule-energy UV sources used so far to generate plasmas with similar parameters $[37,38]$. Such relaxed requirements for laser energy pave the way to the experiments at high repetition rates, which is very promising for applications in laser-driven nuclear physics. For instance, the cross-sections for the nuclear-excitation-by-electroncapture reactions in the hot plasma will be 3-4 orders of magnitude higher compared to the "cold" $\mathrm{x}$-ray freeelectron laser plasma case as reported in Refs. [42,43]. Also, the simulations of the temporal evolution of the $\mathrm{He}_{\alpha}$ and $\mathrm{Ly}_{\alpha}$ emission suggest that ultra-high-temporal contrast, relativistically intense femtosecond laser pulses enable ultrashort ( $\leq 150 \mathrm{fs}$ ) $\mathrm{x}$-ray pulses. Such pulses are very attractive for using them for time-resolved $\mathrm{x}$-ray radiography of high-density plasmas [44].

\section{ACKNOWLEDGMENTS}

We would like to thank N. E. Andreev for fruitful discussions. Financial support has been provided by the Federal Ministry of Education and Research, Germany, Grant No. 05P15SJFA1. S. A., V. S., A. P., and A. B. acknowledge financial support of the Austrian Science Fund (FWF), projects SFB "NextLite" F49-03 and P 27491-N27.

[1] S. Suckewer and P. Jaegle, X-Ray Laser: Past, Present and Future, Laser Phys. Lett. 6, 411 (2009).

[2] V. N. Shlyaptsev, J. J. Rocca, M. Grisham, G. Avaria, and F. Tomasel, Prospective Schemes for Next Generation X-Ray Lasers, Proc. SPIE Int. Soc. Opt. Eng. 7451, 745103 (2009).

[3] R. A. Smith, V. Barrow, J. Edwards, G. Kiehn, and O. Willi, High Density Plasmas for Recombination X-Ray Lasers, Appl. Phys. B 50, 187 (1990).

[4] L. J. Perkins, B. G. Logan, M. D. Rosen, M. D. Perry, T. D. de la Rubia, N. M. Ghoniem, T. Ditmire, P. T. Springer, and S. C. Wilks, The Investigation of High Intensity Laser Driven Micro Neutron Sources for Fusion Materials Research at High Fluence, Nucl. Fusion 40, 1 (2000).

[5] A. Curtis, C. Calvi, J. Tinsley, R. Hollinger, V. Kaymak, A. Pukhov, S. Wang, A. Rockwood, Y. Wang, V. N. Shlyaptsev, and J. J. Rocca, Micro-Scale Fusion in Dense Relativistic Nanowire Array Plasmas, Nat. Commun. 9, 1077 (2018).

[6] S. V. Bulanov, T. Z. Esirkepov, D. Habs, F. Pegoraro, and T. Tajima, Relativistic Laser-Matter Interaction and Relativistic Laboratory Astrophysics, Eur. Phys. J. D 55, 483 (2009).

[7] S. Fujioka, H. Takabe, N. Yamamoto, D. Salzmann, F. Wang, H. Nishimura, Y. Li, Q. Dong, S. Wang, Y. Zhang, Y.-J. Rhee, Y.-W. Lee, J.-M. Han, M. Tanabe, T. Fujiwara, Y. Nakabayashi, G. Zhao, J. Zhang, and K. Mima, X-Ray Astronomy in the Laboratory with a Miniature Compact Object by Laser Driven Implosion, Nat. Phys. 5, 821 (2009).

[8] G. Mourou, Z. Chang, A. Maksimchuk, J. Nees, S. V. Bulanov, V. Yu. Bychenkov, T. Z. Esirkepov, N. M. Naumova, F. Pegoraro, and H. Ruhl, On the Design of Experiments for the Study of Relativistic Nonlinear Optics in the Limit of Single-Cycle Pulse Duration and Single-Wavelength Spot Size, Plasma Phys. Rep. 28, 12 (2002).

[9] M. N. Polyanskiy, M. Babzien, and I. V. Pogorelsky, 100Terawatt $\mathrm{CO}_{2}$ Laser: Design and Current Status, AIP Conf. Proc. 1777, 110006 (2016). 
[10] S. Tochitsky, F. Fiuza, and C. Joshi, Prospects and Directions of $\mathrm{CO}_{2}$ Laser-Driven Accelerators, AIP Conf. Proc. 1777, 020005 (2016).

[11] G. Andriukaitis, T. Balčiūnas, S. Ališauskas, A. Pugžlys, A. Baltuška, T. Popmintchev, M.-C. Chen, M. M. Murnane, and H. C. Kapteyn, 90 GW Peak Power Few-Cycle MidInfrared Pulses from an Optical Parametric Amplifier, Opt. Lett. 36, 2755 (2011).

[12] V. Shumakova, S. Ališauskas, P. Malevich, C. Gollner, A. Baltuška, D. Kartashov, A. M. Zheltikov, A. V. Mitrofanov, A. A. Voronin, D. A. Sidorov-Biryukov, and A. Pugžlys, Filamentation of Mid-IR Pulses in Ambient Air in the Vicinity of Molecular Resonances, Opt. Lett. 43, 2185 (2018).

[13] F. Brunel, Not-So-Resonant, Resonant Absorption, Phys. Rev. Lett. 59, 52 (1987).

[14] P. Gibbon and A. R. Bell, Collisionless Absorption in Sharp-Edged Plasmas, Phys. Rev. Lett. 68, 1535 (1992).

[15] S. C. Wilks and W. L. Kruer, Absorption of Ultrashort, Ultra-Intense Laser Light by Solids and Overdence Plasmas, IEEE J. Quantum Electron. 33, 1954 (1997).

[16] P. Mulser, S.-M. Weng, and T. Liseykina, Analysis of the Brunel Model and Resulting Hot Electron Spectra, Phys. Plasmas 19, 043301 (2012).

[17] T. Liseykina, P. Mulser, and M. Murakami, Collisionless Absorption, Hot Electron Generation and Energy Scaling in Intense Laser-Target Interaction, Phys. Plasmas 22, 033302 (2015).

[18] J. Wu and C. Guo, Wavelength Effects on Strong-Field Single Electron Ionization, Adv. Stud. Theor. Phys. 2, 271 (2008).

[19] J. Weisshaupt, V. Juvé, M. Holtz, S. A. Ku, M. Woerner, T. Elsaesser, S. Ališauskas, A. Pugžlys, and A. Baltuška, HighBrightness Table-Top Hard X-Ray Source Driven by Sub100-Femtosecond Mid-Infrared Pulses, Nat. Photonics 8 , 927 (2014).

[20] J. Weisshaupt, V. Juvé, M. Holtz, M. Woerner, and T. Elsaesser, Theoretical Analysis of Hard X-Ray Generation by Nonperturbative Interaction of Ultrashort Light Pulses with a Metal, Struc. Dyn. 2, 024102 (2015).

[21] A. Pukhov, Three-Dimensional Electromagnetic Relativistic Particle-in-Cell Code VPL (Virtual Laser Plasma Lab), J. Plasma Phys. 61, 425 (1999).

[22] H.-K. Chung, M. H. Chen, W. L. Morgan, Yu. Ralchenko, and R. W. Lee, FLYCHK: Generalized Population Kinetics and Spectral Model for Rapid Spectroscopic Analysis for All Elements, High Energy Density Phys. 1, 3 (2005).

[23] G. Mourou, The Ultrahigh-Peak-Power Laser: Present and Future, Appl. Phys. B 65, 205 (1997).

[24] S. Kroker, T. Käsebier, S. Steiner, E.-B. Kley, and A. Tünnermann, High Efficiency Two-Dimensional Grating Reflectors with Angularly Tunable Polarization Efficiency, Appl. Phys. Lett. 102, 161111 (2013).

[25] C. Hahn, G. Weber, R. Märtin, S. Höfer, T. Kämpfer, and T. Stöhlker, CdTe Timepix Detectors for Single-Photon Spectroscopy and Linear Polarimetry of High-Flux Hard X-Ray Radiation, Rev. Sci. Instrum. 87, 043106 (2016).

[26] P. P. Rajeev, P. Taneja, P. Ayyub, A. S. Sandhu, and G. R. Kumar, Metal Nanoplasmas as Bright Source of Hard X-Ray Pulses, Phys. Rev. Lett. 90, 115002 (2003).
[27] T. Nishikawa, S. Suzuki, Y. Watanabe, O. Zhou, and H. Nakano, Efficient Water-Window X-Ray Pulse Generation from Femtosecond-Laser-Produced Plasma by Using a Carbon Nanotube Targets, Appl. Phys. B 78, 885 (2004).

[28] S. Mondal, I. Chakraborty, S. Ahmad, D. Carvalho, P. Singh, A. D. Lad, V. Narayanan, P. Ayyub, G. R. Kumar, J. Zheng, and Z. M. Sheng, Highly Enhanced Hard X-Ray Emission from Oriented Metal Nanorod Arrays Excited by Intense Femtosecond Laser Pulses, Phys. Rev. B 83, 035408 (2011).

[29] X-Ray Data Booklet, 3rd ed., edited by A. C. Thompson and D. Vaughan (Ernest Orlando Lawrence Berkeley National Laboratory, Berkeley, CA, USA, 2009), pp. 1-16.

[30] M. R. Khan and M. Karimi, $K_{\beta} / K_{\alpha}$ Ratios in EnergyDispersive X-Ray Emission Analysis, X-Ray Spectrom. 9, 32 (1980).

[31] Z. Samsonova, S. Höfer, R. Hollinger, T. Kämpfer, I. Uschmann, R. Röder, L. Trefflich, O. Rosmej, E. Förster, C. Ronning, D. Kartashov, and C. Spielmann, Hard $X$-ray Generation from $\mathrm{ZnO}$ Nanowire Targets in a NonRelativistic Regime of Laser-Solid Interactions, Appl. Sci. 8, 1728 (2018).

[32] G. H. McCall, Calculation of X-Ray Bremsstrahlung and Characteristic Line Emission Produced by a Maxwellian Electron Distribution, J. Phys. D 15, 823 (1982).

[33] A. Macchi, M. Borghesi, and M. Passoni, Ion Acceleration by Superintense Laser-Plasma Interaction, Rev. Mod. Phys. 85, 751 (2013).

[34] M. Roth and M. Schollmeier, Ion Acceleration-Target Normal Sheath Acceleration, edited by B. Holzer (CERN, Geneva, Switzerland, 2016), Vol. 1, p. 231.

[35] M. Sherlock, Universal Scaling of the Electron Distribution Function in One-Dimensional Simulations of Relativistic Laser-Plasma Interactions, Phys. Plasmas 16, 103101 (2009).

[36] O. N. Rosmej, Z. Samsonova, S. Höfer, D. Kartashov, C. Arda, D. Khaghani, A. Schoenlein, S. Zähter, A. Hoffmann, R. Loetzsch, I. Uschmann, M. E. Povarnitsyn, N. E. Andreev, L. P. Pugachev, M. C. Kaluza, and C. Spielmann, Generation of keV Hot Near-Solid Density Plasma States at High Contrast Laser-Matter Interaction, Phys. Plasmas 25, 083103 (2018).

[37] M. A. Purvis, V. N. Shlyaptsev, R. Hollinger, C. Bargsten, A. Pukhov, A. Prieto, Y. Wang, B. M. Luther, L. Yin, S. Wang, and J. J. Rocca, Relativistic Plasma Nanophotonics for Ultrahigh Energy Density Physics, Nat. Photonics 7, 796 (2013).

[38] C. Bargsten, R. Hollinger, M. G. Capeluto, V. Kaymak, A. Pukhov, S. Wang, A. Rockwood, Y. Wang, D. Keiss, R. Tommasini, R. London, J. Park, M. Busquet, M. Klapisch, V. N. Shlyaptsev, and J. J. Rocca, Energy Penetration into Arrays of Aligned Nanowires Irradiated with Relativistic Intensities: Scaling to Terabar Pressures, Sci. Adv. 3, e1601558 (2017).

[39] V. Kaymak, A. Pukhov, V. N. Shlyaptsev, and J. J. Rocca, Nanoscale Ultradense Z-Pinch Formation from LaserIrradiated Nanowire Arrays, Phys. Rev. Lett. 117, 035004 (2016).

[40] A. M. Perelomov, V. S. Popov, and M. V. Terent'ev, Ionization of Atoms in an Alternating Electric Field, Sov. Phys. JETP 23, 924 (1966). 
[41] R. Hollinger, C. Bargsten, V. N. Shlyaptsev, V. Kaymak, A. Pukhov, M. G. Capeluto, S. Wang, A. Rockwood, Y. Wang, A. Townsend, A. Prieto, P. Stockton, A. Curtis, and J. J. Rocca, Efficient Picosecond X-Ray Pulse Generation from Plasmas in the Radiation Dominated Regime, Optica 4, 1344 (2017).

[42] J. Gunst, Y. A. Litvinov, C. H. Keitel, and A. Pálffy, Dominant Secondary Nuclear Photoexcitation with the X-Ray FreeElectron Laser, Phys. Rev. Lett. 112, 082501 (2014).
[43] J. Gunst, Y. Wu, N. Kumar, C. H. Keitel, and A. Pálffy, Direct and Secondary Nuclear Excitation with X-Ray Free-Electron Lasers, Phys. Plasmas 22, 112706 (2015).

[44] A. Morace, L. Fedeli, D. Batani, S. Baton, F. N. Beg, S. Hulin, L. C. Jarrot, A. Margarit, M. Nakai, P. Nakatsutsumi, P. Nicolai, N. Piovella, M. S. Wei, X. Vaisseau, L. Volpe, and J. J. Santos, Development of X-Ray Radiography for High Energy Density Physics, Phys. Plasmas 21, 102712 (2014). 